\title{
A Novel Dual-Polarized Planar Antenna
}

\author{
Hai-han Sun, Can Ding, and Y. Jay Guo \\ Global Big Data Technologies Centre (GBDTC) \\ University of Technology Sydney (UTS) \\ Sydney, NSW, Australia, 2007 \\ Haihan.Sun@student.uts.edu.au
}

\begin{abstract}
A wideband dual-polarized antenna with a novel planar configuration is presented for base station applications. Two groups of simple dipoles are fed by two microstrip feed networks to achieve $\pm 45^{\circ}$ polarizations. A novel feeding technique that leads to a planar configuration is described. Measured results show that excellent matching and stable radiation performances are achieved over a wide band.
\end{abstract}

Keywords-dual-polarization; planar structure; wideband

\section{INTRODUCTION}

Base station antenna (BSA) array is one of the most crucial devices for current and future generation cellular communication systems. It consists of several BSA elements which are required to provide $\pm 45^{\circ}$ polarization with stable radiation patterns across a wide band. Most of the known BSA elements are based on dipoles, including cross-dipoles [1]-[3], magneto-electric dipoles (ME dipoles) [4], and square dipole arrays [5]. To excite a dipole, a Balun is a must to guarantee balanced feed. Balun as part of the feed network is usually placed between the radiators and the ground, occupying a large space. On the other hand, there has been ever growing demand to make base station antennas more compact and highly integrated, especially for $5 \mathrm{G}$ systems. Therefore, it is of great value to integrate the Balun with the antenna and use the saved space between the antenna and the ground plane to locate other devices and electronics such as phase shifters.

Some BSAs with planar structures have been proposed recently [6], [7]. The antenna in [6] has its feed network printed on the same substrate with the radiating dipoles. However, only single polarization has been achieved by such a configuration. In [7], two dual-dipole elements were used to realize a dual-polarized antenna with planar structure. Each dual-dipole are differentially fed by two coaxial cables through a short stub. Wide impedance matching with high isolation and acceptable radiation performances are achieved, but a $180^{\circ}$ hybrid coupler is needed to provide differential feeds, which increases the design complexity.

In this paper, a planar wideband $\pm 45^{\circ}$ dual-polarized BSA with excellent radiation performance is proposed. Four simple dipoles as the radiating elements are fed by two specially designed planar feed networks to realize the two polarizations. With a simple and compact structure, the proposed antenna achieves stable radiation patterns with halfpower-beamwidth (HPBWs) varying within $67^{\circ} \pm 7^{\circ}$, stable gains around $8.5 \mathrm{dBi}$, high port isolations $>29 \mathrm{~dB}$, and good impedance matching with $\left|\mathrm{S}_{11}\right|<-10 \mathrm{~dB}$ across a wide bandwidth of $66.6 \%$.

\section{CONFIGURATION OF THE ANTENNA}

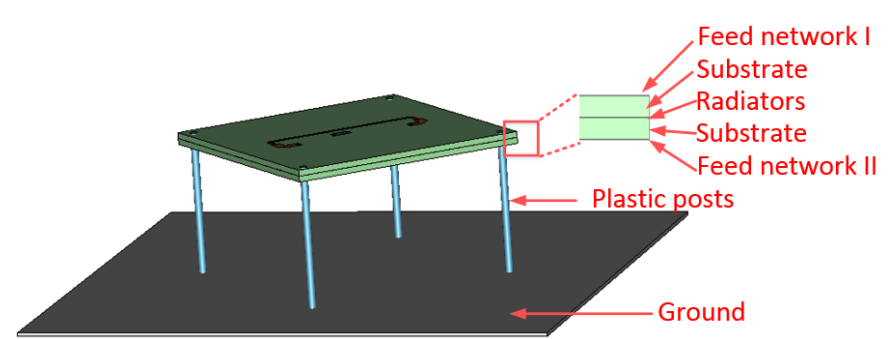

(a)

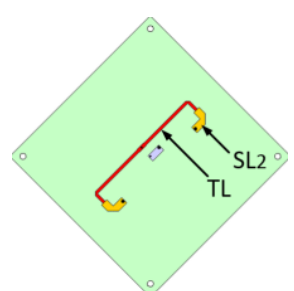

(b)

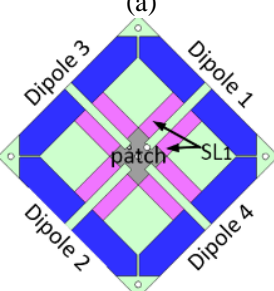

(c)

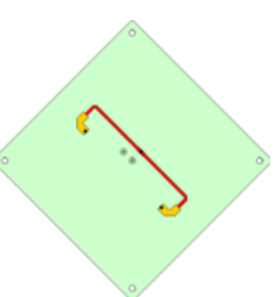

(d)
Fig. 1 Antenna configuration: (a) Perspective view; Top views of the (b) top, (c) middle, and (d) bottom metallic layers.

The configuration of the planar dual-polarized antenna is shown in Fig. 1. It consists of two substrate layers and three metallic layers stacked together. Four dipoles as radiators are located on the middle metallic layer and two feed networks are positioned on the top and bottom metallic layers. Each feed network excites two dipoles simultaneously for one polarization. The stacked layers with radiators and feed networks are placed above a ground plane with a distance of $35 \mathrm{~mm}$ and are supported by four plastic posts. The substrate used in this work is Rogers 4350B with a dielectric constant of 3.48 and a thickness of $1.524 \mathrm{~mm}$.

\section{DESIGN OF THE FEED NETWORK}

The feed networks of the proposed antenna need to excite a pair of dipoles simultaneously while providing good impedance matching. Fig. 2 shows the circuit representation of the proposed feed network. Since the dipoles are identical and have the same input impedance $\mathrm{Z}$, the feed network is a duplication of the matching circuit for one dipole. As shown in Fig. 2, the input impedance of each dipole is firstly transformed to nearly $100 \Omega\left(\mathrm{Z}_{1}{ }^{\prime}, \mathrm{Z}_{2}{ }^{\prime}\right)$ by an impedance matching circuit, and then connected in parallel to match to a standard $50-\Omega$ coaxial cable. 
Impedance matching network

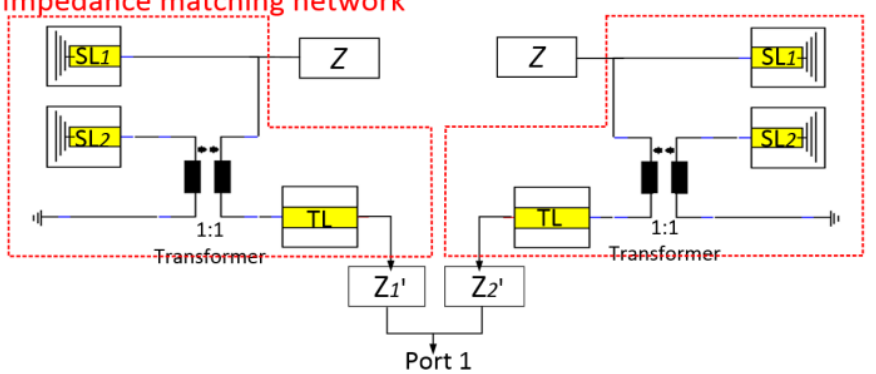

Fig. 2 Circuit representation of the feed network.

The impedance matching circuit for each dipole consists of one transmission line TL and two short lines $\mathrm{SL}_{1}$ and $\mathrm{SL}_{2}$. The matching result with the optimized matching circuit is shown in the result section. In this work, microstrip lines were used for the implementation, as shown in Fig. 1. The $\mathrm{SL}_{1} \mathrm{~S}$ are coupled lines connecting dipole arms functioning as Balun to provide balanced feed. They are also connected to a rectangular patch located at the center of the board for shorting purpose. The dipoles, $\mathrm{SL}_{1} \mathrm{~S}$, and the shorting patch are all located on the same layer. The $\mathrm{SL}_{1} \mathrm{~S}$ with the shorting patch also acts as the ground for the microstrip lines TL and $\mathrm{SL}_{2}$. Their dimensions are calculated based on the optimized results from the circuit optimization in ADS. The antenna is fed by $50-\Omega$ coaxial cables, where the inner conductor connected to the feed lines on the top and bottom layer, and the outer conductor connected to the patch on the middle layer.

\section{Simulated AND MEASUREd RESUlts}

The proposed antenna was also fabricated and tested. Fig. 3 presents the prototype of the antenna. The simulated and measured S-parameters, and the matching result based on circuit theory model are shown in Fig. 4. The measured results agree well with the simulated one. According to the measured results, $\left|\mathrm{S}_{11}\right| /\left|\mathrm{S}_{22}\right|$ are $<-10 \mathrm{~dB}$ across a wide bandwidth of $66.6 \%$ from $1.5 \mathrm{GHz}$ to $3.0 \mathrm{GHz}$. The port-toport isolations are $>29 \mathrm{~dB}$ over this band. The radiation patterns in the horizontal plane at $1.7 \mathrm{GHz}, 2.2 \mathrm{GHz}$, and 2.7 $\mathrm{GHz}$ are shown in Fig. 5. According to the measured radiation patterns, the HPBWs are maintained within $67^{\circ} \pm 7^{\circ}$ and the cross polarization discriminations (XPDs) are $>22$ $\mathrm{dB}$ at boresight for both the two polarizations. Moreover, the measured gains are from $7.3 \mathrm{dBi}$ to $9.2 \mathrm{dBi}$ across the band.

\section{CONCLUSION}

A novel planar $\pm 45^{\circ}$ dual-polarized antenna element comprising four simple dipoles was proposed. The feed networks for this antenna are designed on two layers that are closely attached to the radiation layer, so a large space between the radiation layer and the ground is saved for other purposes. Even under the strict space constraint for the feed network, excellent performance is still achieved. According to the measured results, the proposed antenna has stable HPBWs, high gains, and high XPDs across a wide band from $1.5 \mathrm{GHz}$ to $3.0 \mathrm{GHz}$ with $\left|\mathrm{S}_{11}\right| /\left|\mathrm{S}_{22}\right|<-10 \mathrm{~dB}$, thus serving as a great candidate for $5 \mathrm{G}$ base station antennas.

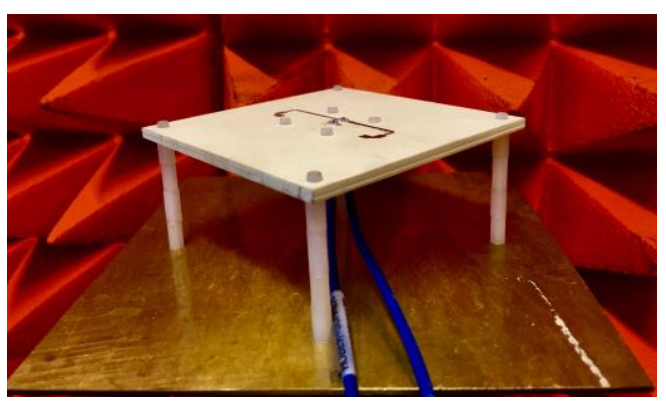

Fig. 3 Prototype of the antenna.

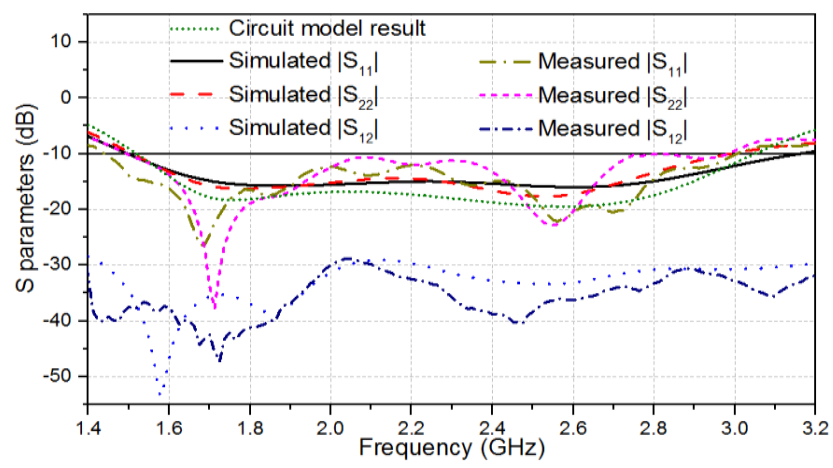

Fig. 4 Circuit matching results, and the simulated and measured $\mathrm{S}$ parameters.

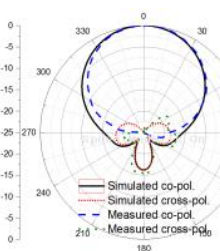

(a)

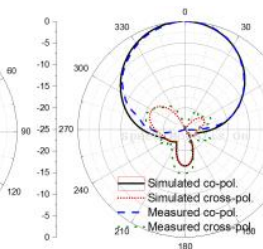

(b)

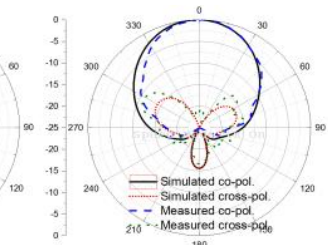

(c)
Fig. 5 Simulated and measured radiation patterns. (a) $1.7 \mathrm{GHz}$, (b) $2.2 \mathrm{GHz}$, (c) $2.7 \mathrm{GHz}$.

\section{REFERENCES}

[1] Z. Bao, Z. Nie, and X. Zong, "A novel broadband dual-polarization antenna utilizing strong mutual coupling," IEEE Trans. Antennas Propag., vol. 62, no. 1, pp. 450-454, Jan. 2014.

[2] H. Huang, Y. Liu, and S. Gong, "A broadband dual-polarized base station antenna with sturdy construction," IEEE Antennas Wireless Propag. lett., vol. 16, pp. 665-668, 2017.

[3] C. Ding, H. Sun, R. W. Ziolkowski and Y. J. Guo, "Simplified tightlycoupled cross-dipole arrangement for base station applications," IEEE Access, vol. 5, pp. 27491-27503, Nov. 2017.

[4] L. Siu, H. Wong, and K. M. Luk, "A dual-polarized magneto-electric dipole with dielectric loading," IEEE Trans. Antennas Propag., vol. 57, no. 3, pp. 616-623, Mar. 2009.

[5] H. Sun, C. Ding, B. Jones and Y. J. Guo, "A wideband base station antenna element with stable radiation pattern and reduced beam squint," IEEE Access, vol. 5, pp. 23022-23031, Oct. 2017.

[6] Y. H. Cui, R. L. Li, P. Wang, "A novel broadband planar antenna for 2G/3G/LTE base stations", IEEE Trans. Antennas Propag., vol. 61, no. 5, pp. 2767-2774, May 2013.

[7] Y. Cui, X. Gao, and R. Li, "A broadband differential fed dualpolarized planar antenna", IEEE Trans. Antennas Propog., vol. 65, iss. 6, pp. 3231-3234, June 2017. 